\title{
Editorial: Generation 1.5 in Canada: Multiple Perspectives on a Shifting Demographic Landscape
}

Welcome to the TESL Canada special themed issue on Generation 1.5. Generation 1.5 has been an emergent profile of learner long recognized in Canada, but not adequately addressed in the research in the Canadian context. Although this topic has been well recognized in the United States, this issue is now becoming timely in Canada. The Canadian context is distinct and requires a response tailored to our local (national) context: one that is different from that of our southern neighbors.

As co-editors of this themed issue, we sought to include perspectives and voices from among all those who work and conduct research with students and their families, including academics, graduate students, teachers, community-based organizations, and ministries of education. The contributions included are wide-ranging and reflect a variety of perspectives and research approaches. The boundaries of the readership of the TESL Canada Journal are expanding to include concerns related to the K-12 experiences of Englishlanguage learners and their subsequent participation in postsecondary studies. For a better understanding of the postsecondary experiences of English-language learners, it becomes important to consider the onset of the Canadian educational trajectory of the demographic broadly identified as Generation 1.5.

The issue begins with a literature review of data-informed research about Generation 1.5. The article highlights patterns of academic achievement and identifies areas of educational concern for students who are not well served by the (K-12 school) system. The article further highlights the reality that Generation 1.5 is not a monolithic construct and that there is great diversity within this demographic.

After an overview of the empirical research on Generation 1.5, the contribution of Cummins, Mirza, and Stille provides a retrospective of Cummins' major theoretical insights and extends into current work on the importance of identity texts and identity construction. The next four contributions (Jean \& Geva; Kim \& Duff; Mossman; and Faez) take up the issue of negotiation of identity and provide qualitative insights into language socialization for young learners, youths, university students, and preservice Generation 1.5 teachers. The final two research articles shift to provide a quantitative focus on data. Gunderson, D'Silva, and Odo use data available from the Vancouver School District and the British Columbia Ministry of Education to track longitudinal outcomes. Leo uses discourse analysis and corpus linguistics 
methodologies to investigate the patterns of Generation 1.5 writing on entrance to university.

Our special issue of the TESL Canada Journal then shifts toward practice in classrooms and in the community. The first contribution (Salmon \& Ettrich) in the Perspectives section describes the K-12 language proficiency benchmark initiative in Alberta. Biemiller follows this by underscoring the role of vocabulary development in the educational success of Generation 1.5. Finally, In the Classroom (and in the community) bridges theory and practice in the community to programming and curricular innovations. Ngo's article captures the perceptions of Generation 1.5 families on culturally responsive practice in the K-12 system. The final two articles by Jasinski and Crossman and Pinchbeck describe curricular interventions for supporting immigrant students and their families.

As co-editors, we are pleased with the contributions, and we make special recognition of the reviewers. We are further grateful to TESL Canada for identifying this topic as one worthy of special attention in a themed issue.

Hetty Roessingh and Scott Roy Douglas 


\section{Éditorial : La génération 1,5 au Canada: perspectives multiples d'un profil démographique en mutation}

Bienvenue au numéro spécial de la Revue TESL du Canada sur le thème de la génération 1,5. La génération 1,5 représente un profil d'apprenants reconnu depuis longtemps au Canada, mais un qui n'a tout de même pas assez fait l'objet de recherche dans le contexte canadien. Ce sujet bien reconnu aux États-Unis prend de l'importance au Canada. Le contexte canadien est distinct et exige une réaction adaptée à sa réalité locale (nationale) et donc très différente de celle de nos voisins au sud.

Comme coéditeurs de ce numéro à thème, nous avons voulu inclure des perspectives de tous ceux qui travaillent et font de la recherche avec les étudiants et leurs familles, y compris les chercheurs universitaires, les étudiants des cycles supérieurs, les organisations communautaires et les ministères de l'éducation. Les articles couvrent tout un éventail de sujets et reflètent une diversité de perspectives et d'approches à la recherche. La gamme des sujets d'intérêt des lecteurs de la Revue TESL du Canada s'élargit pour inclure des enjeux liés aux expériences K-12 des apprenants de l'anglais langue seconde et leur participation subséquente aux études postsecondaires. Afin de mieux comprendre les expériences postsecondaires des apprenants de l'anglais, il devient important de considérer les débuts de la trajectoire éducationnelle canadienne que suit le groupe démographique identifié, au sens large, comme la génération 1,5.

Le numéro débute par une analyse documentaire de la recherche portant sur la génération 1,5. Cet article fait ressortir des modèles de rendement académique et identifie des domaines préoccupants chez les étudiants qui sont mal servis par le système scolaire (K-12). Il souligne également le fait que la génération 1,5 n'est pas une entité monolithique mais plutôt un groupe démographique caractérisé par une grande diversité.

Suite à cet aperçu de la recherche empirique sur la génération 1,5, l'article de Cummins, Mirza et Stille offre une rétrospective des connaissances théoriques majeures de Cummins en plus d'un aperçu du travail actuel sur l'importance des textes identitaires et la construction de l'identité. Les quatre articles suivants (Jean \& Geva, Kim \& Duff, Mossman, et Faez) portent sur le sujet de la négociation de l'identité et offrent des aperçus qualitatifs sur la socialisation linguistique chez les jeunes apprenants, les étudiants à l'université et les stagiaires de la génération 1,5. Les deux derniers articles de recherche changent d'orientation pour se pencher sur des données quantita- 
tives. Gunderson, D'Silva et Odo puisent dans des données du district scolaire de Vancouver et du ministère de l'éducation de la Colombie britannique pour étudier les résultats longitudinaux. Leo a recours à une analyse de discours et à des méthodologies de la linguistique de corpus pour étudier les modèles qu'emploient des étudiants de la génération 1,5 quand ils rédigent des textes pour entrer à l'université.

Notre numéro spécial de la Revue TESL du Canada passe ensuite à la pratique en salle de classe et dans la communauté. Le premier texte (Salmon \& Ettrich) dans la section Perspectives décrit l'initiative portant sur les niveaux de compétence linguistique K-12 en Alberta. Biemiller suit en soulignant l'importance du rôle du développement du vocabulaire dans la réussite scolaire de la génération 1,5. Finalement, la section Dans la salle de classe (et dans la communauté) fait le pont entre la théorie et la pratique dans la communauté relativement à la programmation et aux innovations dans les programmes d'études. L'article de Ngo décrit les perceptions qu'ont les familles de la génération 1,5 des pratiques dans le système $\mathrm{K}-12$ relativement à l'adaptation aux différences culturelles. Les deux derniers articles, un par Jasinski et l'autre par Crossman et Pinchbeck, décrivent les interventions pédagogiques à l'appui des élèves immigrants et leurs familles.

En tant que coéditeurs, nous sommes enchantés du choix des articles, et nous tenons à offrir nos remerciements aux lecteurs critiques. Nous sommes également reconnaissants envers TESL Canada d'avoir identifié ce sujet comme thème pour un numéro spécial.

Hetty Roessingh et Scott Roy Douglas 